\title{
Platelet Rich Fibrin for the Treatment of Cleft like Gingival Recession: A Case Report
}

\author{
Bashar $\mathrm{AKM}^{1 *}$, Rafique $\mathrm{T}^{2}$, Ghosh $\mathrm{R}^{2}$, Biswas $\mathrm{AK}^{2}$, Akter $\mathrm{K}^{1}$, Hassan $\mathrm{GS}^{2}$ \\ ${ }^{\prime}$ Department of Conservative Dentistry \& Endodontics, Bangabandhu Sheikh Mujib Medical University, Dhaka, \\ Bangladesh; ${ }^{2}$ Department of Orthodontics, Bangabandhu Sheikh Mujib Medical University, Dhaka, Bangladesh
}

\begin{abstract}
Background: Gingival recession exposes the tooth's root with aesthetic disfigurement, frequently brings a patient to a dentist's office for the objectionable aesthetics caused by the disease. This case report addressed the management of localized cleft like gingival recession in the tooth no 31 with the aid of Platelet Rich Fibrin (PRF).

Methods: Mucosal flap was retracted from tooth no 41 to 33 followed by curettage and root planning on the effected tooth (where there was gingival recession). Allogenic bone graft was applied over the affected area, covered with freshly prepared autogenous PRF membrane, flap was repositioned and sutured.

Results: Within six month after surgery previously denuded root surface of the treated incisor became entirely covered with mucosa and good aesthetics was regained; which after 4 years, maintained stable gingival contour and aesthetics.

Conclusion: The result in the presented case indicate that PRF can be applied when cleft like gingival recession due to malocclusion is necessarily to be treated uneventfully with good aesthetics.
\end{abstract}

Keywords: Gingival recession, Platelet-rich fibrin

\section{Introduction}

Gum recession commonly manifestedas tooth looking longer than normal, occurs when the edge of the gingival margins moves away from the crown of the tooth. The two main predisposing factors are tooth malposition and tooth to alveolar bone relationship. ${ }^{1}$ If left untreated, the supporting tissue and bone structures of the teeth can be severely damaged, and may ultimately result in tooth loss. Studies have shown that where the shape and position of teeth results in a thin alveolar bone, these areas are predisposed to fenestrations, dehiscence, and gingival recession. ${ }^{2}$ Other aetiologic factors that have been implicated are tooth brushing trauma, mechanical trauma including biting habits, local irritants including plaque and calculus, iatrogenic restorations, associated high frenal attachments and periodontal disease. $^{3}$ However, what frequently brings a patient to a dentist's office is not pain, but rather the objectionable aesthetics caused by the disease.

*Correspondence: AKM Bashar, Department of Conservative Dentistry \& Endodontics, Bangabandhu Sheikh Mujib Medical University, Dhaka, Bangladesh; e-mail: drbasharakm@gmail.com ORCID: https://orcid.org/0000-0003-0353-9418
Treatments typically result in aesthetic improvement, elimination of sensitivity and a decreased risk of developing root caries. ${ }^{4}$ Management of gingival recession may include several methods such as pocket depth reduction, the gingival grafting, Pinhole Surgical Technique (PST ${ }^{\mathrm{TM}}$ ), Guided Tissue Regeneration (GTR) using collagen membrane and more recently with Platelet-rich plasma (PRP) or Platelet-rich fibrin (PRF) to accelerate soft-tissue and hard-tissue healing. $5,6,7,8$

\section{Case presentation}

A nine years old boy, came to the Orthodontics Department of Bangabandhu Sheikh Mujib Medical University (BSMMU), Dhaka with the complaint of compromised esthetics at tooth no 31 along with bleeding during tooth brushing on his clinical examination a cleft like gingival recession that measured $6 \mathrm{~mm}$ with surrounding red festoon seen on tooth no 31. (figure 1). He had mixed dentition with mild crowding. Guided Tissue Regeneration using PRF membrane along with 
allogenic bone graft was planned. Selective grindings of some teeth was carried out to minimize the occlusal prematurity and interferences.

\section{Clinical procedures}

Preparation of PRF: Fifty $\mathrm{ml}$ blood is drawn into five 10-ml test tubes without any anticoagulant and centrifuged immediately at $2,700 \mathrm{rpm}$ for $12 \mathrm{~min}$ using a tabletop centrifugal mechine. ${ }^{9}$ The resultant product consists of the three layers and the PRF clot was formed in the middle layer of the test tube (figure 2). PRF was obtained in the form of a membrane by squeezing out the fluids in the fibrin clot. ${ }^{9}$

Surgical procedure:At first the surgical area of the patient's mouth was prepared using chlorhexidine mouthwash. Bilateral incisive nerve block was given to anesthetize the surgical area.

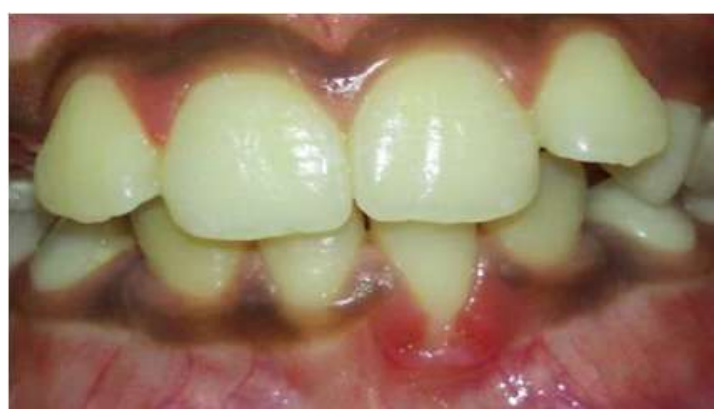

Figure 1: Preoperative figure showing a $6 \mathrm{~mm}$ cleft like gingival defect on tooth no 31

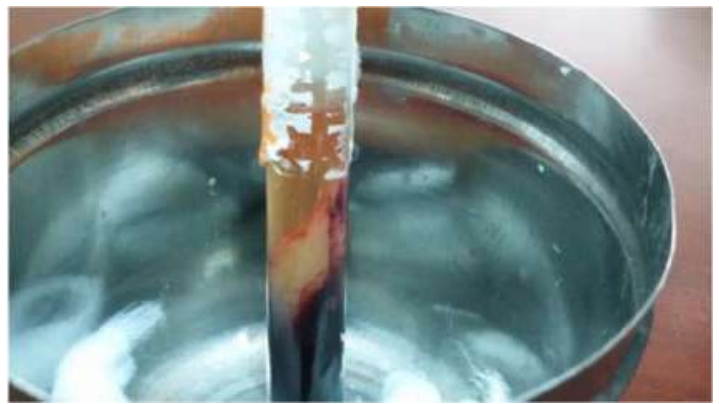

Figure 2: Prepared three layer including middle layer consisting of PRF after centrifuge

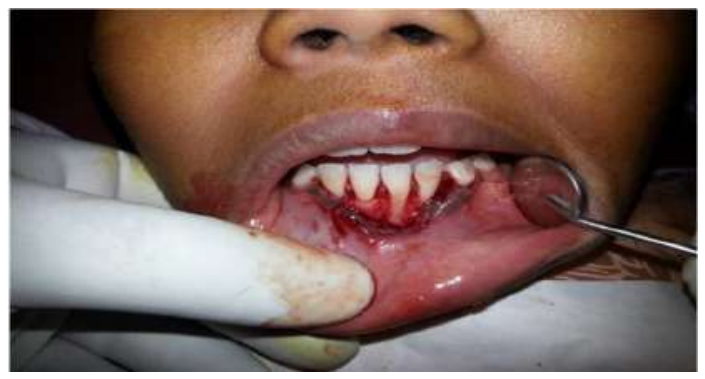

Figure 3: Root planning and curettege done after flap retraction
A crestal incision was made from tooth no 41 to 33, mucosal flap was retracted and curettage - root planning done in the effected tooth no 31 . (figure 3) $0.5 \mathrm{cc}$ allogenic bone graft was applied over the affected area, covered with freshly prepared autogenous PRF. Finally flap repositioned and sutured. (figure 4)

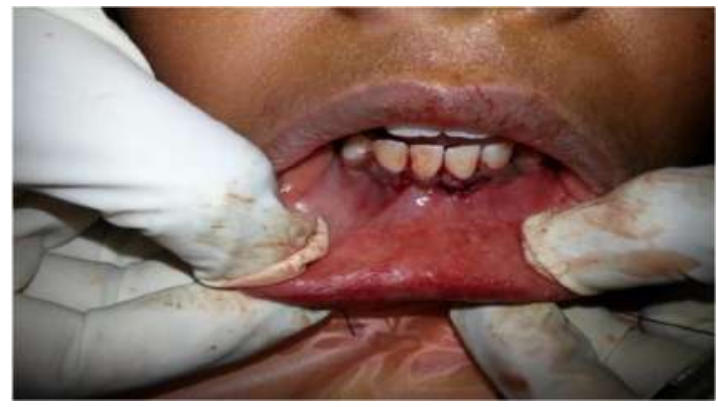

Figure 4: Retracted \& sutured flap immediately after bone graft and PRF placement

\section{Results}

Within six month after surgery impressive attachment had developed, previously denuded root surface of the treated incisor became entirely covered. (figure 5) The marginal tissue felt well attached; probing depth found minimal and good esthetics was retained. A follow up period of 4 years, stable gingival contour and aesthetics maintained. (figure 6)

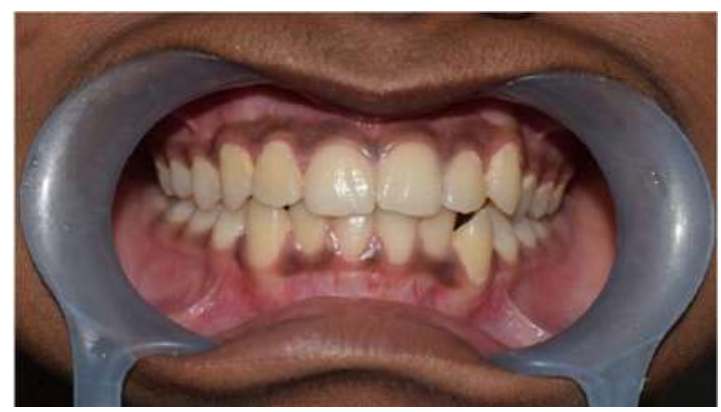

Figure 5: Six months after surgery. Repair of the defect with good esthetics

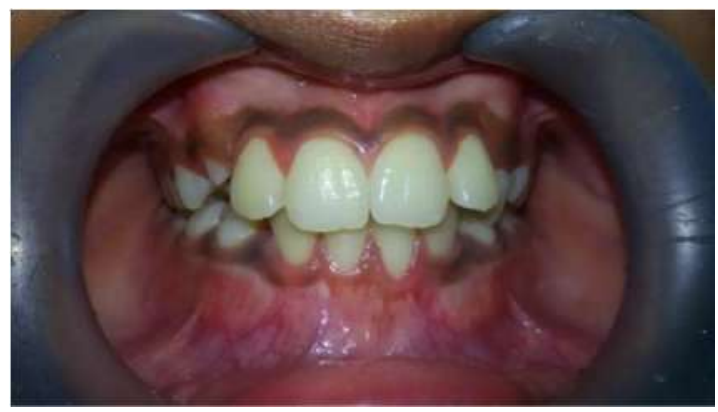

Figure 6 : Four years after Surgery 


\section{Discussion}

PRF described first by Choukrounet al. ${ }^{10}$ has been referred to as a second-generation platelet concentrate, which has been shown to have several advantages over the traditionally used methods. The free gingival graft, most widely used procedure because of using autogenous graft, is very sensitive. Graft's dimensions and border characteristics impact root coverage success while stabilization and immobilization of the graft also has a crucial role. There is increased discomfort and potential for postoperative bleeding from the donor area by virtue of a large wound that heals by secondary intention. ${ }^{5}$ Platelet-Rich Plasma (PRP) is a platelet concentrate that is being used widely to accelerate soft-tissue and hard-tissue healing. Sanchez et al. ${ }^{11}$ have elaborated on the potential risks associated with the use of PRP. The preparation of PRP involves the isolation of PRP from autologous blood, a double centrifugation technique is required after which gel formation is accelerated using calcium chloride and bovine thrombin. It has been discovered that the use of bovine thrombin may be associated with the development of antibodies to the factors V, and XI, resulting in the risk of life-threatening coagulopathies. Bovine thrombin preparations have also been shown to contain factor $\mathrm{V}$, which could result in the stimulation of the immune system when challenged with a foreign protein. But PRF eliminates the redundant process of adding anticoagulant as well as the need to neutralize it. Because of the absence of an anticoagulant, blood begins to coagulate as soon as it comes in contact with the glass surface. Here the conversion of fibrinogen into fibrin takes place slowly with small quantities of physiologically available thrombin present in the blood sample itself. Thus, a physiologic architecture that is very favorable to the healing process is obtained due to this slow polymerization process.

\section{Conclusion}

The result in the presented case indicates that cleft like gingival recession is necessarily to be treated uneventfully with good aesthetics with the application of the PRF with bone graft. However, source of trauma should be eliminated by proper adjustment of the occlusion for stable result as done in this case.

\section{References}

1. Ravipudi S, Appukuttan D, Prakash PSG, Victor DJ. Gingival Recession: Short Literature Review on Etiology, Classifications and Various Treatment Options. J. Pharm. Sci. \& Res. 2017;9:215-20.

2. Sawan NM, Ghoneima A, Stewart K, Liu S. Risk factors contributing to gingival recession among patients undergoing different orthodontic treatment modalities. Interv Med Appl Sci. 2018 Mar;10: 19-26.

DOI: doi.org/10.1556/1646.9.2017.42

3. Jati AS, Furquim LZ, Consolaro A. Gingival recession: its causes and types, and the importance of orthodontic treatment. Dental Press J Orthod. 2016;21:18-29.

DOI:doi.org/10.1590/2177-6709.21.3.018-029.oin

4. Levine, Robert A. Aesthetics in Periodontics: The Subepithelial Connective Tissue Graft for Root Coverage: Report on 20 Teeth in 10 Patients. Compendium of Continuing Education in Dentistry, XII:8:568,1991.

5. Saglam M, Koseoglu S. Treatment of localized gingival recessions with free gingival graft. European Journal of General Dentistry. 2012;1:10-14. DOI: doi.org/10.4103/2278-9626.101347

6. Naik AR, Alampalli V, Ramesh AV, Dwarkanath CD, Naik MS, Chinnappa AB. Use of autologous platelet rich plasma to treat gingival recession in esthetic periodontal surgery. J Indian Soc Periodontol. 2013;17:345-53.

DOI: doi.org/10.4103/0972-124X.115665

7. Sampoornam S. Pinhole Surgical Technique for treatment of marginal tissue recession: A case series. J Indian Soc Periodontol. 2017; 21:507-11.

8. Anilkumar K, Geetha A, Umasudhakar, Ramakrishnan T, Vijayalakshmi R, Pameela E. Platelet-rich-fibrin: A novel root coverage approach. $J$ Indian Soc Periodontol. 2009; 13:50-54.

DOI: doi.org/10.4103/0972-124X.51897

9. Sunitha Raja, Munirathnam Naidu E. Platelet rich fibrin: Evolution of a secondgeneration platelet concentrate. Indian J Dent Res. 2008;19:42-6. DOI: doi.org/10.4103/0970-9290.38931

10. Choukroun J, Diss A, Simonpieri A, Girard MO, Schoeffler C, Dohan SL, et al. Platelet-rich fibrin (PRF): A second generation platelet concentrate: Part I: Technological concepts and evolution. Oral Surg Oral Med Oral Pathol Oral RadiolEndod 2006;101:E37-44.

DOI:doi.org/10.1016/j.tripleo.2005.07.011

11. Sanchez AR, Sheridan PJ, Kupp LI. Is platelet-rich plasma the perfect enhancement factor? A current review. Int J Oral Maxillofac Implants 2003;18:93103. 